\title{
Correction to: Nonuniform Discrete Wavelets on Local Fields of Positive Characteristic
}

\section{Younus Bhat ${ }^{1}$}

Published online: 30 July 2018

(c) Springer Nature Switzerland AG 2018

\section{Correction to: Complex Anal. Oper. Theory https://doi.org/10.1007/s11785-018-0813-6}

In the original publication, the text of Sects. 1 and 2 up to Definition 2.3 follows closely the text published in Sects. 1, 2 and 3 up to Definition 3.2 of the article "Nonuniform Multiresolution Analysis on Local Fields of Positive Characteristic" by F.A. Shah and Abdullah, published in Complex Analysis and Operator Theory 9 (2015), pp. 1589-1594 (cited as Ref. [15]). We apologise for having missed to mention this.

The original article can be found online at https://doi.org/10.1007/s11785-018-0813-6.

$\varangle \quad$ M. Younus Bhat gyounusg@gmail.com

1 Department of Mathematics, Islamic University of Science and Technology, Awantipora, Jammu and Kashmir 192122, India 